\title{
Ascorbic Acid Improves Productivity, Physio Biochemical Attributes and Antioxidant Activity of Deficit Irrigated Broccoli Plants
}

\author{
Ashraf Sh Osman ${ }^{1}$, Mohamed H Abdel Wahed ${ }^{2}$ and Mostafa M Rady*3 \\ ${ }^{1}$ Horticulture, Department, Fayoum University, Egypt
}

${ }^{2}$ Soils and water Science Department, Fayoum University, Egypt

${ }^{3}$ Botany Department, Fayoum University, Egypt

Received: 輼: October 25, 2018; Published: 制: November 13, 2018

*Corresponding author: Mostafa M Rady, Soils and Water Science Department, Egypt

\begin{abstract}
Introduction: Ascorbic acid (AsA) acts as an enzyme co-factor, an antioxidant, and a donor/acceptor in electron transport at the plasma membrane or in the chloroplasts. It controls plant growth and induces drought tolerance in plants through maintaining tissue turgor.

Aim: Two experiments were conducted in 2013/2014 and 2014/2015 to study the effect of 1 or 2 mM AsA on growth, productivity and WUE, concentrations of leaf pigments, osmo protectants and endogenous AsA, and DPPH radical-scavenging activity of broccoli plants grown under three levels of irrigation ( $\mathrm{DI}_{0 \%}=100 \%, \mathrm{DI}_{20 \%}=80 \%$ and $\mathrm{DI}_{40 \%}=60 \%$ of ETC).

Results: All above parameters significantly affected by DI and the combined DI+ AsA applications. The combined DI+ AsA treatment positively modified the negative effects of DI and improved all the aforesaid parameters. The combined treatment of $\mathrm{DI}_{20 \%}+1$ or 2 mM AsA produced plants having growth, yields and WUE higher than or as those of the plants generated under full irrigation.

Conclusion: we conclude that with exogenous 1 or $2 \mathrm{mM}$ AsA application, the DI20\% strategy could be successfully applied for the production of commercial broccoli allowing water savings of at least $20 \%$ without any detrimental effects on broccoli performances.

Keywords: Deficit Irrigation, Ascorbic Acid, Antioxidant Activity, Broccoli Performance

Abbreviations: Asa: Ascorbic Acid; DI: Deficit Irrigation; WUE: Water Use Efficiency; ROS: Reactive Oxygen Species; $\mathrm{O}_{2}$ : Superoxide; O: Singlet Oxygen; $\mathrm{H}_{2} \mathrm{O}_{2}$ : Hydrogen Peroxide; Oh: Hydroxyl Anion; IWA: Irrigation Water Applied; ETC: Crop Evapotranspiration/Crop Water Requirements; FM: Fresh Weight; TM: Turgid Weight; DW: Dry Weight; RWC: Relative Water Content; MSI: Membrane Stability Index
\end{abstract}

\section{Introduction}

Broccoli (Brassica oleracea L. var. italica) is an important winter vegetable crop due to its nutritional and medicinal values. It is a rich source of health promoting phytochemicals, and it contains high records of vitamins, minerals, and antioxidants that prevent the formation of cancer-causing agents [1,2]. It is widely cultivated in many European and American countries [3] but it is still not well known and is grown in very limited scattered areas in Egypt. For good performance with higher quality, broccoli requires a sufficient water supply [4], depending on soil type, location and its conditions, and growth stage.

It requires approx. 3 to 4 million liters of water either by drip, sprinkler or furrow irrigation [5], while the available amount of water for agriculture is declining worldwide [6]. Under optimal water supply, the highest crop productivity is achieved for highyielding varieties, but under limited water supply crops will adapt to water stress and can produce well FAO [7]. As an on farm strategy for dry regions, DI may provide water savings in some cases with negligible lost in yield [8] through successful management of the limited amount of water, depending on better agricultural practices and enhanced understandings of water productivity [9]. DI is used as a strategy for water-savings [10] in which water reduction during a particular period or throughout the whole growing season must be considered. The main goal of this strategy is to increase crop WUE either by reducing the amount of water applied or by reducing the number of irrigation events [11]. 
It is well known that water scarcity stress increases the production of ROS such as $\mathrm{O}_{2}^{-}, \mathrm{H}_{2} \mathrm{O}_{2},{ }^{1} \mathrm{O}_{2}$ and $\mathrm{OH}^{-}$radicals in chloroplasts, peroxisomes and mitochondria. These ROS affect, negatively, various physiological processes and plant growth [12] and death of cells will occur if stress is prolonged [13]. Application of water stressed plants with some antioxidants such as As A will help crop plants to tolerate water scarcity stress. AsA $\left(\mathrm{C}_{6} \mathrm{H}_{8} \mathrm{O}_{6}\right)$ is a small molecular weight powerful antioxidant. With helping of the enzyme ascorbate peroxidase, it scavenges and controls the concentration of $\mathrm{H}_{2} \mathrm{O}_{2}$ in plants [14]. It participates in a variety of processes including photosynthesis, cell wall growth and cell expansion, and increases plant resistance to water deficit stress $[15,16]$. Therefore, the current study was aimed to assess the efficacy of AsA foliar application at a rate of 1 or $2 \mathrm{mM}$ under the application of DI strategy (60, 80 and $100 \%$ of ET $)$ in two growing seasons on WUE, growth and productivity, and physio-biochemical attributes in broccoli as a model plant.

\section{Methodology}

\section{Experimental Site and Procedures}

Table 1: Physical and chemical characteristics of the experimental site for 2013/2014 and 2014/2015 seasons.

\begin{tabular}{|c|c|c|}
\hline Property & $2013 / 2014$ & $2014 / 2015$ \\
\hline \multicolumn{3}{|l|}{ Physical properties: } \\
\hline Clay \% & 49.47 & 48.21 \\
\hline Slit \% & 21.61 & 22.75 \\
\hline Fine sand \% & 23.17 & 23.11 \\
\hline Coarse sand \% & 5.75 & 5.93 \\
\hline Soil Texture & clay & clay \\
\hline \multicolumn{3}{|l|}{ Chemical properties } \\
\hline $\mathrm{P}^{\mathrm{H}}(1: 25)$ & 7.41 & 7.48 \\
\hline CEC(meq/100g soil) & 37.22 & 36.86 \\
\hline $\operatorname{ECe}\left(\mathrm{ds} \mathrm{m}^{-1}\right)$ & 2.83 & 2.88 \\
\hline Organic matter (\%) & 1.81 & 1.73 \\
\hline $\mathrm{CaCo}_{3}(\%)$ & 4.31 & 4.23 \\
\hline $\mathrm{N}(\%)$ & 0.59 & 0.56 \\
\hline \multicolumn{3}{|c|}{$\begin{array}{l}\text { Available elements }\left(\mathrm{mg} \mathrm{kg}^{-1}\right. \\
\text { soil): }\end{array}$} \\
\hline $\mathrm{K}$ & 652 & 703 \\
\hline $\mathrm{P}$ & 562 & 523 \\
\hline $\mathrm{Zn}$ & 18 & 19 \\
\hline $\mathrm{Cu}$ & 0.74 & 0.77 \\
\hline $\mathrm{Fe}$ & 0.64 & 0.66 \\
\hline Mn & 6.75 & 70.1 \\
\hline \multicolumn{3}{|c|}{ Soluble Cations (m mole $\mathrm{L}^{-1}$ ) } \\
\hline $\mathrm{K}^{+}$ & 0.69 & 0.74 \\
\hline $\mathrm{Ca}^{++}$ & 14.21 & 14.98 \\
\hline $\mathrm{Mg}^{++}$ & 3.52 & 3.48 \\
\hline $\mathrm{Na}^{+}$ & 10.01 & 10.35 \\
\hline $\mathrm{HCO}^{-3}$ & 0.26 & 0.27 \\
\hline $\mathrm{Cl}^{-}$ & 1.36 & 1.23 \\
\hline $\mathrm{So}^{-4}$ & 5.02 & 4.86 \\
\hline
\end{tabular}

Two field experiments were conducted during 2013/2014 and 2014/2015 seasons in a private Farm, Fayoum province, Egypt. Before transplanting, soil physical and chemical analyses were performed according to standard procedures $[17,18]$ and the results are presented in Table 1. Broccoli hybrid seeds cv. Groene Calabrese (Seminis-Peto seed Company, USA) were sown in the nursery on mid of September, 2013 and 2014. In each season, 30 -d-old seedlings were transplanted into the field on $40 \mathrm{~cm}$ spaces on rows. Each experimental unit was planned to cover an area of $12 \mathrm{~m}^{2}$ including five rows of $4 \mathrm{~m}$ long and $0.6 \mathrm{~m}$ wide. The experimental layout was a factorial arranged as a split-plot with three replications.

During soil preparations for transplanting, all experimental areas were received the complete doses of different fertilizers (40 unit of $\mathrm{P}_{2} \mathrm{O}_{5}, 80$ unit of $\mathrm{N}$ and 60 unit of $\mathrm{K}_{2} \mathrm{O}$ ha ${ }^{-1}$ ) that were side banded at two equal portions; 3 and 6 weeks after transplanting. The other recommended Agro-management practices were performed. Irrigation water levels were randomly occupied main plots that isolated with $2 \mathrm{~m}$ fallow land to prevent borders effects, while AsA rates (0, 1 or $2 \mathrm{mM}$ ) were randomly allowed for subplots, which were foliar sprayed three times, to run off; 30, 40 and $50 \mathrm{~d}$ after transplanting. The IWA were done in three treatments; $\mathrm{DI}_{0 \%}=$ irrigation with $100 \%, \mathrm{DI}_{20 \%}=$ irrigation with $80 \%$, and $\mathrm{DI}_{40 \%}=$ irrigation with $60 \%$ of the crop water requirements (ET). The daily $\mathrm{ET}_{\mathrm{o}}$ was computed by the following equation Doorenbos et al. [11]

$$
\text { ETo }=\text { Kpan } \times \text { Epan }
$$

where, $\mathrm{E}_{\mathrm{pan}}$ is the evaporation from the Class A pan $\left(\mathrm{mm} \mathrm{d}^{-1}\right)$ and $\mathrm{K}_{\mathrm{pan}}$ is the pan evaporation coefficient.

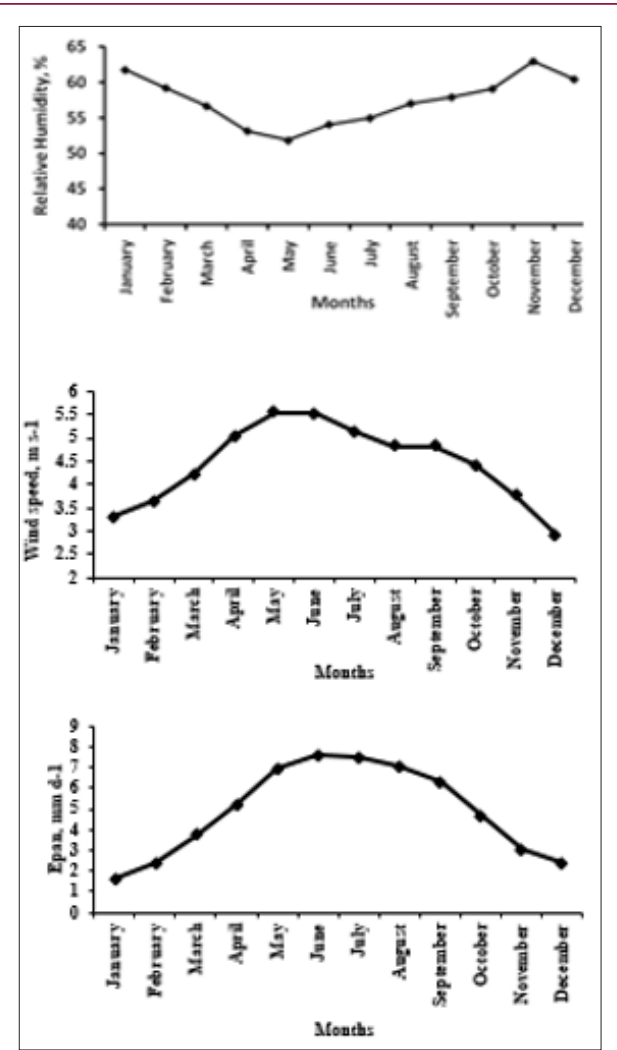

Figure 1: Monthly mean relative humidity, wind speed and class A pan evaporation from Itsa Station. 
Monthly mean weather data for years of this study (January 2013 - December 2015) were obtained from the weather station, Fayoum province and are shown in Figure 1. The average daily $\mathrm{E}_{\mathrm{pan}}$ was $7.65,7.51,7.07$ and $6.33 \mathrm{~mm} \mathrm{~d}^{-1}$ for October, November, and December, respectively. The ETc were estimated using the crop coefficient according to the following equation:

$$
E T c=E T o \times K c
$$

where, $\mathrm{ET}_{\mathrm{c}}$ is the crop water requirements, $\mathrm{mm} \mathrm{d}^{-1}$ and $\mathrm{K}_{\mathrm{c}}$ is the crop coefficient.

The lengths of the different crop growth stages were 35, 45, 40 and 15 days for initial, crop development, mid-season and late season, respectively. The crop coefficients $\left(\mathrm{K}_{\mathrm{c}}\right)$ of initial, mid and end stages were $0.70,1.05$ and 0.95 , respectively according to Allen et al. [7]. Broccoli plants were irrigated at 15-d-interval by different amounts of water. The amount of IWA for each plot was determined by using the equation given below:

$$
\mathrm{IWA}=\frac{\mathrm{A} \times \mathrm{ETc} \times \mathrm{I}}{\mathrm{E} \times 1000}+\mathrm{R}
$$

where, IWA is the irrigation water applied $\left(\mathrm{m}^{3}\right)$, A is the plot area $\left(\mathrm{m}^{2}\right)$, ETc is the crop water requirements $\left(\mathrm{mm} \mathrm{d}^{-1}\right)$, Ii is the irrigation intervals (d), $\mathrm{E}_{\mathrm{a}}$ is the application efficiency (\%), and LR is the leaching requirements $\left(\mathrm{m}^{3}\right)$. Amount of IWA was controlled through plastic pipe (spiles) of $50 \mathrm{~mm}$ diameter. One spile per plot was used to convey water for each plot and amount of water delivered through a plastic pipe was calculated using the following equation [19].

$$
Q=C A \sqrt{2 g h^{*} 10^{-3}}
$$

where, $\mathrm{Q}$ is the discharge of irrigation water $\left(\mathrm{l}_{\mathrm{sec}} \mathrm{se}^{-1}\right), \mathrm{C}$ is the coefficient of discharge, $\mathrm{A}$ is the cross section area of irrigation pipe $\left(\mathrm{cm}^{2}\right), \mathrm{g}$ is the gravity acceleration $\left(\mathrm{cm} . \mathrm{sec}^{-2}\right)$, and $\mathrm{h}$ is the average effective head of water $(\mathrm{cm})$.

\section{Plant Sampling and Measurements of Morphological Characteristics and Yields}

In each experimental unit, plants of the two outer rows were allocated for morphological characteristics and physio-biochemical measurements, while the three inner rows were left to head yields and their components, and WUE. Leaf samples from three randomly selected 70-d-old plants were collected and washed for some determinations, and others were dried at $70^{\circ} \mathrm{C}$ in a forced-air oven till constant weight. When flowering reached approx. 25\% (10 weeks after transplanting), growth features were taken using three randomly selected plants from each experimental unit. Plants were carefully cut off at the ground level and immediately carried to the laboratory where they were separated into leaf-blades and stems including leaf-petioles. Stem length $(\mathrm{cm})$ was measured beginning from the ground level to the apical meristem. Numbers of branches and leaves plant ${ }^{-1}$ were counted. Total leaf area plant ${ }^{-1}\left(\mathrm{~m}^{2}\right)$ was calculated using leaf area/leaf weight relationship [20]. Shoot dry weight plant ${ }^{-1}(\mathrm{~g})$ was taken after oven drying at $70^{\circ} \mathrm{C}$ in a forced-air oven till constant weights.

In each experimental plot, all plants of the three middle rows were allocated to head yields and their components. Central and lateral heads having closed floral buds, dark green color and good compactness were harvested, and stalks, then were trimmed. Diameter of central head $(\mathrm{cm})$ was measured at ground level by using Sealy So707-Digital Electronic Vernier Caliper 0-150 mm/0$6 "$. Number of lateral heads was counted. Average weight of central, lateral, and total heads plant ${ }^{-1}$ (g) and ha-1 (metric tonnes) was taken during the whole harvesting period.

\section{Measurements of RWC, MSI and WUE}

Fully-expanded leaf discs were used to assess the RWC\% Hayat et al., [21] with modification of Osman et al. [22]. Discs FM were taken and immediately immersed in double-distilled water for $24 \mathrm{~h}$, in the dark, to saturate them. TM was measured after gently drying of adhering water. Discs were then dehydrated at $70^{\circ} \mathrm{C}$ until constant weight to record DM and the following formula was used:

$$
R W C(\%)=\left[\frac{(F M-D M)}{(T M-D M)}\right] * 100
$$

Duplicate $0.2 \mathrm{~g}$ samples of fully-expanded leaf tissues were used to assess MSI Rady [23]. A sample was placed in test-tube containing double-distilled water and heated at $40^{\circ} \mathrm{C}$ for $30 \mathrm{~min}$. Electrical conductivity $\left(\mathrm{C}_{1}\right)$ of the solution was recorded. The second sample was boiled at $100^{\circ} \mathrm{C}$ for $10 \mathrm{~min}$ and the $\mathrm{C}_{2}$ was measured, and the following formula was used:

$$
\operatorname{MSI}(\%)=\left[1-\left(\frac{C 1}{C 2}\right)\right]^{*} 100
$$

Values of WUE (kg heads $\mathrm{m}^{-3}$ IWA) were calculated for different treatments after harvest according to the following equation [24]. WUE $=$ heads yield $\left(M\right.$ ton ha $\left.{ }^{-1}\right) /$ water applied $\left(\mathrm{m}^{3} \mathrm{ha}^{-1}\right)$

\section{Determination of Leaf Pigments}

Leaf chlorophyll and carotenoid concentrations (mg g ${ }^{-1}$ fresh weight) were assessed according to Arnon [25], after homogenization in $80 \%(\mathrm{v} / \mathrm{v})$ acetone and centrifugation at 10,000 $\times \mathrm{g}$ for $10 \mathrm{~min}$. The absorbance of acetone extract was measured at 663,645 , and $470 \mathrm{~nm}$ using a UV-visible spectrophotometer (Bausch and Lomb-2000 Spectronic 21). Anthocyanin concentration (mg $\mathrm{g}^{-1}$ dry weight) was determined using the $\mathrm{pH}$ differential method Meyers et al., [26] by a spectrophotometer at wavelengths of 510 $\mathrm{nm}$ and $700 \mathrm{~nm}$ using buffers from potassium chloride $\left(\mathrm{P}^{\mathrm{H}} 1.0\right)$ and sodium acetate $\left(\mathrm{P}^{\mathrm{H}} 4.5\right)$, where $\mathrm{A}=\left(\mathrm{A}_{510}-\mathrm{A}_{700}\right)$

$$
\mathrm{P}^{\mathrm{H}} 1-\left(\mathrm{A}_{510}-\mathrm{A}_{700}\right) \mathrm{P}^{\mathrm{H}} 4.5 .
$$

\section{Free Proline, Total Soluble Sugars and DPPH -Radical Scavenging Activity Determinations}

Method of Bates et al. [27] was used to determine leaf free proline concentrations, after extraction in $10 \mathrm{ml} 3 \%(\mathrm{v} / \mathrm{v})$ sulphosalicylic acid and centrifugation at $10,000 \times \mathrm{g}$ for $10 \mathrm{~min}$. A $2 \mathrm{ml}$ of the supernatant was received $2 \mathrm{ml}$ of freshly prepared acidninhydrin solution. Mixtures were incubated in a water-bath at $90^{\circ} \mathrm{C}$ for $30 \mathrm{~min}$. The reaction was terminated in an ice-bath and the mixture was extracted again by $5 \mathrm{ml}$ toluene and vortex-mixed for $15 \mathrm{~s}$. After standing for $20 \mathrm{~min}$ in the dark at room temperature to separate phases, toluene phase was carefully collected and the absorbance was read at $520 \mathrm{~nm}$. Method of Irigoyen et al. [28] was used to determine the concentrations of total soluble sugars in 
leaves, after extraction in $96 \%$ ethanol and centrifugation at $3500 \times$ $\mathrm{g}$ for $10 \mathrm{~min}$. Supernatant was collected and stored at $4{ }^{\circ} \mathrm{C}$ and then $3 \mathrm{ml}$ freshly prepared anthrone was added.

Mixtures were incubated in hot water bath for $10 \mathrm{~min}$. The absorbance was recorded at $625 \mathrm{~nm}$ using a UV-160A UV Visible Recording Spectrometer, Shimadzu, Japan. Antioxidant activity was measured using 1,1-diphenyl-2-picrylhydrazyl (DPPH)-radical scavenging activity assay Lee et al., [29]. Stock reagent solution (1 $\times 10^{-3} \mathrm{~mol} \mathrm{l}^{-1}$ ) was prepared by dissolving $22 \mathrm{mg}$ of DPPH in $50 \mathrm{ml}$ methanol and stored at $-20^{\circ} \mathrm{C}$ until use. Working solution $\left(6 \times 10^{-5}\right.$ moll $^{-1}$ ) was prepared by mixing $6 \mathrm{ml}$ of the stock solution with $100 \mathrm{ml}$ methanol to obtain an absorbance value of $0.8 \pm 0.02$ at $515 \mathrm{~nm}$, as measured using a spectrophotometer. Extract solutions of different concentrations $(0.1 \mathrm{ml}$ of each) were vortex-mixed for $30 \mathrm{~s}$ with $3.9 \mathrm{ml}$ of DPPH solution and left to react for $30 \mathrm{~min}$. Absorbance at $515 \mathrm{~nm}$ was recorded. A control with no added extract was also analyzed. Scavenging activity was calculated as follows:

DPPH-radical scavenging activity $(\%)=\left[\left(\mathrm{A}_{\text {control }}-\mathrm{A}_{\text {sample }}\right) /\right.$ $\left.\left(A_{\text {control }}\right)\right] \times 100$ where $A$ is the absorbance at $515 \mathrm{~nm}$.

\section{Determinations of Head Asa Concentrations}

Concentrations of AsA (mg g-1 head FW) were determined using the 2,6-dichloro-indophenol method Helrich [30]. Frozen head samples $(0.2 \mathrm{~g} \mathrm{FW})$ were pulverised in a domestic grinder (Magefesa, Madrid, Spain) and triplicate $10 \mathrm{~g}$ aliquots of each sample were immediately homogenized in $50 \mathrm{ml}$ of $3 \%(\mathrm{w} / \mathrm{v})$ metaphosphoric acid $+8 \%(\mathrm{v} / \mathrm{v})$ acetic acid solution. Extracts were centrifuged at 7,000 $\times \mathrm{g}$ for $15 \mathrm{~min}$, filtered, and made up to 100 $\mathrm{ml}$ with the same homogenization solution. Triplicate aliquots of each sample were titrated with $250 \mathrm{mg} \mathrm{l}^{-1}$ 2,6-dichloro-indophenol solution. The AsA reduced 2,6-dichloro-indophenol to colorless solution and the slight excess of unreduced dye, resulting in lightpink color, indicated the end point of the reaction.

\section{Statistical Analysis}

All data were subjected to analysis of variance for a split-plot design. Significant differences between means were compared at $P \leq 0.05$ using Duncan's multiple range test. The statistical analysis was carried out using COSTAT computer software (Co Hort Software version 6.303, Berkeley, CA, USA).

\section{Results}

Data in Tables 2-4 show that DI affected, negatively, broccoli growth characteristics (i.e., stem length, numbers of leaves and branches plant ${ }^{-1}$, leaf area plant ${ }^{-1}$, and shoot dry weight plant ${ }^{-1}$ ), and yield characteristics (i.e., central and lateral heads weights, and total heads yields plant ${ }^{-1}$ and $\mathrm{ha}^{-1}$ in addition to diameter of central head and No. of lateral heads plant ${ }^{-1}$ ) over 2013/2014 and 2014/2015 growing seasons. Increasing DI from $20 \%$ to $40 \%$ further decreased all growth and yield characteristics. Exogenous application of AsA at a rate of 1 or $2 \mathrm{mM}$ significantly improved all growth and yield characteristics in both growing seasons, particularly under $\mathrm{DI}_{20 \%}$. Although maximum values of growth parameters were obtained with the AsA $+\mathrm{DI}_{0 \%}$ or $\mathrm{DI}_{20 \%}$ treatment, use of 1 or $2 \mathrm{mM}$ AsA for broccoli plants grown under $\mathrm{DI}_{40 \%}$ enabled plants to generate growth characteristics more than or in similar with the plants grown under full irrigation conditions ( $\mathrm{DI}_{0 \%}$ treatment) in both seasons. However, application of 1 or $2 \mathrm{mM}$ AsA for plants grown under $\mathrm{DI}_{40 \%}$ generated lower yield characteristics compared to AsA $+\mathrm{DI}_{0 \%}$ or $\mathrm{DI}_{20 \%}$ treatments in both growing seasons.

Table 2: Effect of exogenous ascorbic acid (AsA) application on growth characteristics of broccoli (Brassica oleracea L. var. italica) plants grown under deficit irrigation.

\begin{tabular}{|c|c|c|c|c|c|c|}
\hline Treatment & & Stem length $(\mathrm{cm})$ & $\begin{array}{c}\text { No. of branches } \\
\text { plant }^{-1}\end{array}$ & $\begin{array}{l}\text { No. of Leaves } \\
\text { plant }^{-1}\end{array}$ & $\begin{array}{l}\text { Leaves area } \\
\text { plant }^{-1}\left(\mathrm{~m}^{2}\right)\end{array}$ & $\begin{array}{c}\text { Shoot dry weight } \\
\text { (g) }\end{array}$ \\
\hline $2013 / 2014$ & season & & & & & \\
\hline \multirow{3}{*}{$\mathrm{DI}_{40 \%}$} & 0 & $31.7 \mathrm{~cd}$ & $8.3 \mathrm{abc}$ & $13.3 \mathrm{~d}$ & $1.79 \mathrm{c}$ & $201 d$ \\
\hline & 1 & $33.2 \mathrm{bcd}$ & 7.9abc & $15.2 \mathrm{bc}$ & $1.89 \mathrm{bc}$ & $202 d$ \\
\hline & 2 & $34.3 \mathrm{bc}$ & $6.8 c$ & $15.6 a b$ & $1.86 \mathrm{bc}$ & $198 d$ \\
\hline \multirow{3}{*}{$\mathrm{DI}_{20 \%}$} & 0 & $33.2 \mathrm{bcd}$ & $7.5 b c$ & $13.8 \mathrm{~cd}$ & $1.91 \mathrm{bc}$ & $236 \mathrm{bc}$ \\
\hline & 1 & $38.3 a$ & $9.2 \mathrm{a}$ & $17.0 \mathrm{a}$ & $2.30 \mathrm{a}$ & $267 a$ \\
\hline & 2 & $41.1 \mathrm{a}$ & $9.1 \mathrm{a}$ & $17.0 \mathrm{a}$ & $2.18 \mathrm{ab}$ & $252 \mathrm{ab}$ \\
\hline \multirow{3}{*}{$\mathrm{DI}_{0 \%}$} & 0 & $31.3 \mathrm{~d}$ & $7.5 \mathrm{bc}$ & $13.7 \mathrm{~cd}$ & $1.92 \mathrm{bc}$ & $223 c$ \\
\hline & 1 & $35.3 \mathrm{~b}$ & $7.7 \mathrm{abc}$ & $14.1 \mathrm{bcd}$ & $2.37 \mathrm{a}$ & $260 a$ \\
\hline & 2 & $34.6 \mathrm{bc}$ & $9.1 \mathrm{a}$ & $14.2 \mathrm{bcd}$ & $2.41 \mathrm{a}$ & $261 \mathrm{a}$ \\
\hline $2014 / 2015$ & season & & & & & \\
\hline \multirow{3}{*}{$\mathrm{DI}_{40 \%}$} & 0 & $35.9 \mathrm{e}$ & $9.4 \mathrm{a}$ & $15.2 \mathrm{~b}$ & $1.72 b$ & $209 d$ \\
\hline & 1 & $38.5 \mathrm{cde}$ & $8.9 \mathrm{ab}$ & $15.1 \mathrm{~b}$ & $1.85 \mathrm{~b}$ & $208 d$ \\
\hline & 2 & $41.3 \mathrm{bc}$ & $6.4 c$ & $15.3 \mathrm{~b}$ & $1.86 \mathrm{~b}$ & $209 d$ \\
\hline \multirow{3}{*}{$\mathrm{DI}_{20 \%}$} & 0 & $39.9 \mathrm{~cd}$ & $8.6 a b$ & $15.9 \mathrm{~b}$ & $1.95 b$ & $248 b c$ \\
\hline & 1 & $44.7 \mathrm{ab}$ & $9.4 \mathrm{a}$ & $18.8 \mathrm{a}$ & $2.33 \mathrm{a}$ & $287 a$ \\
\hline & 2 & $45.7 \mathrm{a}$ & $8.6 \mathrm{ab}$ & $18.2 \mathrm{a}$ & $2.21 \mathrm{a}$ & $265 \mathrm{abc}$ \\
\hline
\end{tabular}




\begin{tabular}{|c|c|c|c|c|c|c|}
\multirow{2}{*}{$\mathrm{DI}_{0 \%}$} & 0 & $36.9 \mathrm{de}$ & $7.6 \mathrm{bc}$ & $15.7 \mathrm{~b}$ & $1.93 \mathrm{~b}$ & $239 \mathrm{c}$ \\
\cline { 2 - 7 } & 1 & $42.2 \mathrm{abc}$ & $8.4 \mathrm{ab}$ & $15.8 \mathrm{~b}$ & $2.37 \mathrm{a}$ & $272 \mathrm{ab}$ \\
\cline { 2 - 7 } & 2 & $39.3 \mathrm{cde}$ & $9.6 \mathrm{a}$ & $16.3 \mathrm{~b}$ & $2.35 \mathrm{a}$ & $280 \mathrm{a}$ \\
\hline
\end{tabular}

Note: Mean values $(n=9)$ in the same column for each trait followed by the same lower-case letter are not significantly different according to Duncan's multiple range test at $\mathrm{P} \leq 0.05 . \mathrm{DI}_{0 \%}=$ irrigation with $100 \%$ of ETc, $\mathrm{DI}_{20 \%}=$ irrigation with $80 \%$ of ETc, and $\mathrm{DI} \mathrm{I}_{40 \%}=$ irrigation with $60 \%$ of ETc.

Table 3: Effect of exogenous ascorbic acid (AsA) application on plant yield and its components of broccoli (Brassica oleracea L. var. italica) plants grown under deficit irrigation.

\begin{tabular}{|c|c|c|c|c|c|c|}
\hline \multicolumn{2}{|c|}{ Treatment } & \multirow{2}{*}{$\begin{array}{c}\text { Diameter of } \\
\text { central head }(\mathrm{cm})\end{array}$} & \multirow{2}{*}{$\begin{array}{l}\text { No. of lateral } \\
\text { heads plant }^{-1}\end{array}$} & \multirow{2}{*}{$\begin{array}{c}\text { Central head } \\
\text { weight plant }^{-1}(\mathrm{~g})\end{array}$} & \multirow{2}{*}{$\begin{array}{c}\text { Lateral head } \\
\text { weight plant }^{-1}(\mathrm{~g})\end{array}$} & \multirow{2}{*}{$\begin{array}{l}\text { Total heads yield } \\
\text { plant }^{-1}(\mathrm{~g})\end{array}$} \\
\hline Irrigation & AsA (mM) & & & & & \\
\hline $2013 / 2014$ & season & & & & & \\
\hline \multirow{3}{*}{$\mathrm{DI}_{40 \%}$} & 0 & $9.3 \mathrm{~cd}$ & $4.0 \mathrm{c}$ & $150 \mathrm{c}$ & $119 \mathrm{c}$ & $269 d$ \\
\hline & 1 & $8.3 d$ & $4.7 \mathrm{bc}$ & $126 \mathrm{~d}$ & $138 \mathrm{c}$ & $263 d$ \\
\hline & 2 & $8.0 \mathrm{~d}$ & $4.2 \mathrm{c}$ & $142 \mathrm{~cd}$ & $132 \mathrm{c}$ & $273 d$ \\
\hline \multirow{3}{*}{$\mathrm{DI}_{20 \%}$} & 0 & $10.0 \mathrm{bc}$ & $5.5 \mathrm{ab}$ & $143 \mathrm{~cd}$ & 214ab & $356 \mathrm{c}$ \\
\hline & 1 & $11.4 \mathrm{ab}$ & $5.7 \mathrm{a}$ & $174 \mathrm{ab}$ & $227 \mathrm{ab}$ & $401 \mathrm{a}$ \\
\hline & 2 & $11.3 \mathrm{ab}$ & $5.3 \mathrm{ab}$ & $181 \mathrm{a}$ & $233 a$ & $415 a$ \\
\hline \multirow{3}{*}{$\mathrm{DI}_{0 \%}$} & 0 & 11.0ab & $5.7 \mathrm{a}$ & $157 \mathrm{bc}$ & $207 \mathrm{~b}$ & $364 \mathrm{bc}$ \\
\hline & 1 & $11.8 \mathrm{a}$ & $5.7 \mathrm{a}$ & $184 a$ & $233 a$ & $417 a$ \\
\hline & 2 & 11.1ab & $6.0 \mathrm{a}$ & $176 a$ & $222 \mathrm{ab}$ & 398ab \\
\hline $2014 / 2015$ & season & & & & & \\
\hline \multirow{3}{*}{$\mathrm{DI}_{40 \%}$} & 0 & $8.3 \mathrm{bc}$ & $4.3 \mathrm{~b}$ & $133 c$ & $128 \mathrm{~d}$ & $262 d$ \\
\hline & 1 & $7.6 \mathrm{c}$ & $4.2 \mathrm{~b}$ & $133 c$ & $137 \mathrm{~cd}$ & $270 \mathrm{~d}$ \\
\hline & 2 & $8.3 \mathrm{bc}$ & $4.2 \mathrm{~b}$ & $135 b c$ & $155 \mathrm{c}$ & $290 \mathrm{~d}$ \\
\hline \multirow{3}{*}{$\mathrm{DI}_{20 \%}$} & 0 & $9.1 \mathrm{ab}$ & $5.2 \mathrm{a}$ & $142 \mathrm{~b}$ & $187 \mathrm{~b}$ & $328 \mathrm{c}$ \\
\hline & 1 & $9.5 \mathrm{a}$ & $5.7 \mathrm{a}$ & $158 \mathrm{a}$ & $216 a$ & $373 a$ \\
\hline & 2 & $9.6 \mathrm{a}$ & $5.3 a$ & 158a & $207 \mathrm{ab}$ & $365 \mathrm{ab}$ \\
\hline \multirow{3}{*}{$\mathrm{DI}_{0 \%}$} & 0 & $9.8 \mathrm{a}$ & $5.5 \mathrm{a}$ & $140 \mathrm{bc}$ & $201 \mathrm{ab}$ & $341 \mathrm{bc}$ \\
\hline & 1 & $10.1 \mathrm{a}$ & $5.5 \mathrm{a}$ & $158 \mathrm{a}$ & $223 a$ & $381 \mathrm{a}$ \\
\hline & 2 & $9.7 \mathrm{a}$ & $5.7 \mathrm{a}$ & $160 \mathrm{a}$ & $207 \mathrm{ab}$ & $367 \mathrm{ab}$ \\
\hline
\end{tabular}

Note: Mean values in the same column for each trait followed by the same lower-case letter are not significantly different according to Duncan's multiple range test at $\mathrm{P} \leq 0.05 . \mathrm{DI}_{0 \%}=$ irrigation with $100 \%$ of ETc, $\mathrm{DI}_{20 \%}=$ irrigation with $80 \%$ of $\mathrm{ETc}_{\text {, and } \mathrm{DI}}=$ irrigation with $60 \%$ of ETc.

Table 4: Effect of exogenous ascorbic acid (AsA) application on hectare yield and its components, and water use efficiency (WUE) of broccoli (Brassica oleracea L. var. italica) plants grown under deficit irrigation.

\begin{tabular}{|c|c|c|c|c|c|}
\hline \multicolumn{2}{|c|}{ Treatment } & \multirow{2}{*}{$\begin{array}{l}\text { Central head weight ha-1 } \\
\text { (metric tonnes) }\end{array}$} & \multirow{2}{*}{$\begin{array}{l}\text { Lateral head weight ha-1 } \\
\text { (metric tonnes) }\end{array}$} & \multirow{2}{*}{$\begin{array}{l}\text { Total heads yield ha- } \\
\text { (metric tonnes) }\end{array}$} & \multirow{2}{*}{$\begin{array}{c}\text { WUE } \\
\text { (kg heads } \mathrm{m}^{-3} \text { IWA) }\end{array}$} \\
\hline Irrigation & AsA (mM) & & & & \\
\hline $2013 / 2014$ & season & & & & \\
\hline \multirow{3}{*}{$\mathrm{DI}_{40 \%}$} & 0 & $6.09 c$ & $4.83 c$ & $10.92 \mathrm{c}$ & $3.81 \mathrm{~cd}$ \\
\hline & 1 & $5.11 \mathrm{~d}$ & $5.59 c$ & $10.71 \mathrm{c}$ & $3.86 \mathrm{bc}$ \\
\hline & 2 & $5.76 \mathrm{~cd}$ & $5.35 \mathrm{c}$ & $11.11 \mathrm{c}$ & 3.88abc \\
\hline \multirow{3}{*}{$\mathrm{DI}_{20 \%}$} & 0 & $5.78 \mathrm{~cd}$ & $8.68 \mathrm{ab}$ & $14.46 \mathrm{~b}$ & $3.78 \mathrm{~cd}$ \\
\hline & 1 & 7.10ab & $9.23 \mathrm{ab}$ & $16.30 \mathrm{a}$ & 4.13ab \\
\hline & 2 & $7.36 \mathrm{a}$ & $9.49 a$ & $16.85 \mathrm{a}$ & $4.17 \mathrm{a}$ \\
\hline \multirow{3}{*}{$\mathrm{DI}_{0 \%}$} & 0 & $6.38 \mathrm{bc}$ & $8.41 \mathrm{~b}$ & $14.79 b$ & $3.10 \mathrm{f}$ \\
\hline & 1 & $7.48 \mathrm{a}$ & $9.49 a$ & $16.97 a$ & $3.55 \mathrm{de}$ \\
\hline & 2 & $7.17 \mathrm{a}$ & $9.08 \mathrm{ab}$ & $16.25 \mathrm{a}$ & $3.40 \mathrm{e}$ \\
\hline
\end{tabular}




\begin{tabular}{|c|c|c|c|c|c|}
\hline $2014 / 2015$ & season & & & & \\
\hline \multirow{3}{*}{$\mathrm{DI}_{40 \%}$} & 0 & $5.43 \mathrm{~b}$ & $5.21 \mathrm{~d}$ & $10.64 \mathrm{e}$ & $3.71 b c$ \\
\hline & 1 & $5.40 \mathrm{~b}$ & $5.57 \mathrm{~cd}$ & $10.97 \mathrm{de}$ & $3.83 \mathrm{ab}$ \\
\hline & 2 & $5.50 \mathrm{~b}$ & $6.29 c$ & $11.78 \mathrm{~d}$ & $4.11 \mathrm{a}$ \\
\hline \multirow{3}{*}{$\mathrm{DI}_{20 \%}$} & 0 & $5.76 \mathrm{~b}$ & $7.58 \mathrm{~b}$ & $13.34 \mathrm{c}$ & $3.52 \mathrm{~cd}$ \\
\hline & 1 & $6.41 \mathrm{a}$ & $8.77 a$ & $15.18 \mathrm{a}$ & 3.97ab \\
\hline & 2 & $6.43 a$ & $8.41 \mathrm{ab}$ & $14.84 \mathrm{ab}$ & $3.91 \mathrm{ab}$ \\
\hline \multirow{3}{*}{$\mathrm{DI}_{0 \%}$} & 0 & $5.69 \mathrm{~b}$ & $8.17 \mathrm{ab}$ & $13.86 b c$ & $2.90 \mathrm{f}$ \\
\hline & 1 & 6.43a & $9.08 \mathrm{a}$ & $15.49 a$ & $3.24 \mathrm{de}$ \\
\hline & 2 & $6.48 a$ & $8.41 \mathrm{ab}$ & 14.89ab & $3.12 \mathrm{ef}$ \\
\hline
\end{tabular}

Note: Mean values in the same column for each trait followed by the same lower-case letter are not significantly different according to Duncan's multiple range test at $\mathrm{P} \leq 0.05$. $\mathrm{D}_{0 \%}=$ irrigation with $100 \%$ of ETc, $\mathrm{DI}_{20 \%}=$ irrigation with $80 \%$ of ETc, and DI $40 \%=$ irrigation with $60 \%$ of ETc.

Data presented in Tables 4 \& 5 show that RWC and MSI of leaf tissues reduced, but WUE increased gradually with increase in DI in both growing seasons. Exogenous AsA application positively modified the DI-affected RWC, MSI, and WUE. Best results for these parameters were obtained from the AsA $+\mathrm{DI}_{0 \%}$ or $\mathrm{DI}_{20 \%}$ treatment. However, AsA $+\mathrm{DI}_{20 \%}$ treatment generated maximum values of WUE in both seasons. Tables $5 \& 6$ show increased concentrations of total chlorophylls, total carotenoids, anthocyanin, free proline, total soluble sugars and AsA, while exhibit decreased antioxidant activity (DPPH-radical scavenging) in broccoli leaves as a result of DI over two growing seasons. Regardless DPPH activity that further reduced with DI from $20-40 \%$, and AsA and carotenoids concentrations that increased with $\mathrm{DI}_{20 \%}$ then reduced under $\mathrm{DI}_{40 \%}$, other photosynthetic pigments and osmoprotectants were increased gradually with the gradual increase in DI over both growing seasons. However, exogenous AsA application at a rate of 1 or $2 \mathrm{mM}$ further increased all photosynthetic pigments, osmoprotectants and endogenous AsA concentrations, and also increased the antioxidant activity (DPPH), particularly under $\mathrm{DI}_{20 \%}$ in both growing seasons.

Table 5: Effect of exogenous ascorbic acid (AsA) application on leaf concentrations of photosynthetic pigments, relative water content (RWC), and membrane stability index (MSI) of broccoli (Brassica oleracea L. var. italica) plants grown under deficit irrigation.

\begin{tabular}{|c|c|c|c|c|c|}
\hline \multicolumn{2}{|c|}{ Treatment } & \multirow{2}{*}{$\begin{array}{c}\text { Free proline } \\
\left(\mu \mathrm{g} \mathrm{g}^{-1} \mathrm{DW}\right)\end{array}$} & \multirow{2}{*}{$\begin{array}{l}\text { Total soluble sugars } \\
\left.\text { ( } \mathrm{mg} \mathrm{g}^{-1} \mathrm{DW}\right)\end{array}$} & \multirow{2}{*}{$\begin{array}{c}\text { Ascorbic acid } \\
\left(\mathrm{mg}^{100 \mathrm{~g}^{-1}} \text { head FW) }\right.\end{array}$} & \multirow{2}{*}{$\begin{array}{c}\text { DPPH radical-scavenging } \\
\text { activity (\%) }\end{array}$} \\
\hline Irrigation & AsA (mM) & & & & \\
\hline $2013 / 2014$ & season & & & & \\
\hline \multirow{3}{*}{$\mathrm{DI}_{40 \%}$} & 0 & 29.9ab & $3.18 \mathrm{a}$ & $78.2 \mathrm{e}$ & $26.8 \mathrm{~d}$ \\
\hline & 1 & $32.6 a$ & $3.23 a$ & $105.3 \mathrm{~b}$ & $29.7 d$ \\
\hline & 2 & $32.9 a$ & $3.33 \mathrm{a}$ & $119.9 a$ & $31.4 \mathrm{~d}$ \\
\hline \multirow{3}{*}{$\mathrm{DI}_{20 \%}$} & 0 & $21.2 \mathrm{de}$ & $2.37 \mathrm{bc}$ & $84.7 \mathrm{de}$ & $38.5 c$ \\
\hline & 1 & $24.6 \mathrm{~cd}$ & $2.56 \mathrm{~b}$ & $98.0 \mathrm{bc}$ & $59.5 a$ \\
\hline & 2 & $25.6 \mathrm{bc}$ & $2.62 b$ & $107.2 \mathrm{~b}$ & $47.6 \mathrm{~b}$ \\
\hline \multirow{3}{*}{$\mathrm{DI}_{0 \%}$} & 0 & $16.6 \mathrm{ef}$ & $2.27 \mathrm{bc}$ & 83.9de & $44.5 \mathrm{~b}$ \\
\hline & 1 & $15.6 \mathrm{f}$ & $2.15 b c$ & $93.2 \mathrm{~cd}$ & $54.2 \mathrm{a}$ \\
\hline & 2 & $14.6 \mathrm{f}$ & $2.05 c$ & $99.0 \mathrm{bc}$ & $46.3 b$ \\
\hline $2014 / 2015$ & season & & & & \\
\hline \multirow{3}{*}{$\mathrm{DI}_{40 \%}$} & 0 & $29.0 \mathrm{ab}$ & $3.59 \mathrm{a}$ & $78.5 \mathrm{~d}$ & $22.2 \mathrm{f}$ \\
\hline & 1 & $31.3 \mathrm{a}$ & $3.55 \mathrm{a}$ & $103.4 \mathrm{bc}$ & $24.5 \mathrm{ef}$ \\
\hline & 2 & $31.9 a$ & $3.51 \mathrm{a}$ & $125.4 \mathrm{a}$ & $26.1 \mathrm{ef}$ \\
\hline \multirow{3}{*}{$\mathrm{DI}_{20 \%}$} & 0 & $19.5 \mathrm{~cd}$ & 2.81a & $84.2 \mathrm{~d}$ & $32.4 \mathrm{de}$ \\
\hline & 1 & $22.9 \mathrm{~cd}$ & $2.67 \mathrm{a}$ & $96.5 \mathrm{c}$ & $60.7 \mathrm{a}$ \\
\hline & 2 & $23.3 \mathrm{bc}$ & $2.49 b c$ & $108.1 \mathrm{~b}$ & $49.3 \mathrm{bc}$ \\
\hline \multirow{3}{*}{$\mathrm{DI}_{0 \%}$} & 0 & $17.4 \mathrm{~cd}$ & $2.23 \mathrm{~cd}$ & $83.7 \mathrm{~d}$ & $41.3 \mathrm{~cd}$ \\
\hline & 1 & $17.3 \mathrm{~cd}$ & $2.19 \mathrm{~cd}$ & $95.1 \mathrm{c}$ & $56.5 \mathrm{ab}$ \\
\hline & 2 & $17.0 \mathrm{~d}$ & $2.12 \mathrm{~d}$ & $101.5 \mathrm{bc}$ & $41.7 \mathrm{c}$ \\
\hline
\end{tabular}

Note: Mean values $(n=9)$ in the same column for each trait followed by the same lower-case letter are not significantly different according to Duncan's multiple range test at $\mathrm{P} \leq 0.05 . \mathrm{DI}_{0 \%}=$ irrigation with $100 \%$ of $\mathrm{ETc}, \mathrm{DI}_{20 \%}=$ irrigation with $80 \%$ of $\mathrm{ETc}$, and $\mathrm{DI}{ }_{40 \%}=$ irrigation with $60 \%$ of ETc. 
Table 6: Effect of exogenous ascorbic acid (AsA) application on hectare yield and its components, and water use efficiency (WUE) of broccoli (Brassica oleracea L. var. italica) plants grown under deficit irrigation.

\begin{tabular}{|c|c|c|c|c|c|c|}
\hline \multicolumn{2}{|c|}{ Treatment } & \multirow{2}{*}{$\begin{array}{l}\text { Total Chlorophylls } \\
\text { (mg g } \text { }^{-1} \text { FW) }\end{array}$} & \multirow{2}{*}{$\begin{array}{c}\text { Total } \\
\text { Carotenoids } \\
\left(\mathrm{mg} \mathrm{g}^{-1} \mathrm{FW}\right)\end{array}$} & \multirow{2}{*}{$\begin{array}{c}\text { Anthocyanin } \\
\left(\mathrm{mg} \mathrm{g}^{-1} \mathrm{DW}\right)\end{array}$} & \multirow{2}{*}{ RWC\% } & \multirow{2}{*}{ MSI $\%$} \\
\hline Irrigation & AsA (mM) & & & & & \\
\hline \multicolumn{7}{|c|}{ 2013/2014 season } \\
\hline \multirow{3}{*}{$\mathrm{DI}_{40 \%}$} & 0 & $2.21 \mathrm{a}$ & $0.29 c$ & $0.54 \mathrm{bc}$ & $72.3 \mathrm{e}$ & $80.9 \mathrm{f}$ \\
\hline & 1 & $2.11 \mathrm{ab}$ & $0.32 \mathrm{c}$ & $0.58 \mathrm{ab}$ & $75.4 \mathrm{de}$ & 82.3ef \\
\hline & 2 & $2.03 \mathrm{~b}$ & $0.31 \mathrm{c}$ & $0.60 \mathrm{a}$ & $79.7 \mathrm{c}$ & $83.3 \mathrm{e}$ \\
\hline \multirow{3}{*}{$\mathrm{DI}_{20 \%}$} & 0 & $1.70 \mathrm{de}$ & $0.33 b c$ & $0.47 \mathrm{e}$ & $78.4 \mathrm{~cd}$ & $86.3 d$ \\
\hline & 1 & $1.78 \mathrm{~cd}$ & $0.37 \mathrm{ab}$ & $0.52 \mathrm{~cd}$ & $84.6 \mathrm{ab}$ & $89.0 \mathrm{bc}$ \\
\hline & 2 & $1.89 \mathrm{c}$ & $0.39 \mathrm{a}$ & $0.55 b c$ & $84.2 \mathrm{ab}$ & $90.5 b$ \\
\hline \multirow{3}{*}{$\mathrm{DI}_{0 \%}$} & 0 & $1.59 \mathrm{e}$ & $0.32 \mathrm{c}$ & $0.41 \mathrm{f}$ & $81.9 b c$ & $87.4 \mathrm{~cd}$ \\
\hline & 1 & $1.69 \mathrm{de}$ & $0.30 \mathrm{c}$ & $0.46 \mathrm{e}$ & $86.5 a$ & $90.8 \mathrm{~b}$ \\
\hline & 2 & $1.78 \mathrm{~cd}$ & $0.32 \mathrm{c}$ & $0.48 \mathrm{de}$ & $88.5 a$ & $92.6 a$ \\
\hline $2014 / 2015$ & Season & & & & & \\
\hline \multirow{3}{*}{$\mathrm{DI}_{40 \%}$} & 0 & $2.06 a$ & 0.23 bcde & $0.57 \mathrm{~b}$ & $75.4 \mathrm{~d}$ & $82.0 \mathrm{e}$ \\
\hline & 1 & $1.92 \mathrm{ab}$ & 0.22 cde & $0.61 \mathrm{a}$ & $77.2 \mathrm{~cd}$ & $84.0 \mathrm{de}$ \\
\hline & 2 & $1.84 \mathrm{bc}$ & $0.20 \mathrm{e}$ & $0.63 a$ & $77.8 \mathrm{~cd}$ & $85.3 \mathrm{~cd}$ \\
\hline \multirow{3}{*}{$\mathrm{DI}_{20 \%}$} & 0 & $1.66 \mathrm{cde}$ & $0.25 \mathrm{bc}$ & $0.48 \mathrm{~d}$ & $78.4 \mathrm{~cd}$ & $87.5 \mathrm{bc}$ \\
\hline & 1 & $1.73 \mathrm{bcd}$ & $0.26 \mathrm{~b}$ & $0.53 c$ & $82.5 \mathrm{abc}$ & $90.0 \mathrm{ab}$ \\
\hline & 2 & $1.83 \mathrm{bc}$ & $0.30 \mathrm{a}$ & $0.57 \mathrm{~b}$ & $85.2 \mathrm{ab}$ & $91.0 \mathrm{a}$ \\
\hline \multirow{3}{*}{$\mathrm{DI}_{0 \%}$} & 0 & $1.50 \mathrm{e}$ & $0.21 \mathrm{de}$ & $0.43 \mathrm{e}$ & $81.0 \mathrm{bcd}$ & $90.2 \mathrm{ab}$ \\
\hline & 1 & $1.57 \mathrm{de}$ & $0.24 \mathrm{bcd}$ & $0.46 \mathrm{de}$ & $86.7 \mathrm{ab}$ & $91.4 \mathrm{a}$ \\
\hline & 2 & $1.61 \mathrm{de}$ & $0.24 \mathrm{bcd}$ & $0.49 \mathrm{~d}$ & $88.1 \mathrm{a}$ & $92.4 \mathrm{a}$ \\
\hline
\end{tabular}

Note: Mean values $(n=9)$ in the same column for each trait followed by the same lower-case letter are not significantly different according to Duncan's multiple range test at $\mathrm{P} \leq 0.05 . \mathrm{DI}_{0 \%}=$ irrigation with $100 \%$ of $\mathrm{ETc}, \mathrm{DI}_{20 \%}=$ irrigation with $80 \%$ of $\mathrm{ETc}$, and $\mathrm{DI} \mathrm{I}_{40 \%}=$ irrigation with $60 \%$ of ETc.

\section{Discussion}

DI is a strategy that could be applied through different irrigation types. DI is sometimes referred to an incomplete supplemental irrigation or regulated DI. A correct DI application requires overall understanding of crop response to water (sensitivity to drought stress), and economic impact of harvest reductions English, et al. [31]. In dry regions (water resources are limited), it can be more fruitful for producers to maximize crop water productivity instead of harvest maximization per unit land Fereres, et al. [8]. The saved water, by application of DI strategy, can be used for other purposes or to irrigate extra lands. Application of a DI strategy put crop plants under water stress (drought), adversely affecting plant growth and physiological processes Loutfy et al. [32]. It has been reported that responses of crop plants to drought depend on species and genotype, length and severity of water deficit, age and development stage, and perhaps on exogenous application of antioxidants for stressed plants Abd El-Mageed, et al. [1].

AsA is one of the non-enzymatic antioxidant compounds. It possesses intrinsic antioxidant properties, serving as electron donors to directly reduce the accumulation of ROS and as reaction substrate within the enzymatic cycle Mittler [33]. It also plays roles in stress signaling and other physiological functions under stress Athar, et al, [34]. Under drought, exogenous applications of AsA have shown to improve plant performance and relieve oxidative stress Khan, et al. [35] that, partially, agreed with our results. Although, efforts have been made to study the addition of AsA for plants to promote plant growth under environmental stresses Dolatabadian et al. [15] there is little information available on the effect of exogenous AsA on plant growth under drought conditions.

In the present study, the exogenous application of AsA found to help to minimize the deleterious effects of DI stress in broccoli plants. It was noticed in this study that DI significantly decreased growth characteristics of broccoli plants (Table 2) which was observed earlier Abd El-Mageed, et al. [10] that may be attributed to a severe damage in plant growth under drought, particularly under $60 \%$ of ETc. In a report, Boutraa [36] reported that water stress conditions cause a multitude of molecular, biochemical and physiological changes, adversely affecting plant growth and development. The decline in plant growth in response to DI stress might be attributed to reductions in cell elongation due to inhibition of growth-promoting hormones, leading to decrease in cell turgor, volume and eventually growth. Water stress conditions cause a marked suppression in plant photosynthetic efficiency, mainly due to stomata closure and Rubisco inhibition Lawlor, et al. [37]. In addition, drought stress leads to an increase in abscisic acid, inhibiting plant growth Abdalla, et al. [38]. A secondary aspect 
of drought stress in plants is the stress-induced production of ROS Azadeh, et al. [39]. Some reports are found to support our results on broccoli plants Zaicovski, et al. [40,41] However, foliar application of AsA found to increase broccoli growth traits under DI stress (Table 2) that was similarly observed earlier Malik, et al. [42].

The beneficial effect of AsA on studied morphological characters may be attributed to its auxinic effect and its protection of plant cells against ROS that are responsible for plant senescence Elade [43]. It may, also, regulate cell wall expansion, cell division, and cell elongation through its action in cell vacuolization Navas, et al. [44]. It improves the absorption of phenolic compounds that protect growing tissues from toxic effects of oxidized phenols Gupta, et al. [45]. In addition, AsA enhances biosynthesis of free amino acids and leaf nitrogen content, which are vital steps in stepping up plant tissues. Under stress, AsA content tends to increase in plants to play a crucial role in regulation of mechanisms of photosynthesis and defense against oxidative stress Dolatabadian, et al. [15]. In this study, the increase in broccoli growth by AsA application under DI stress may be attributed to the increase of water absorption to maintain cell turgor through increase of RWC and MSI (Table 5), accompanying with increase of the concentrations of osmoprotectants, endogenous AsA and DPPH-radical scavenging activity (Table 6) as compared to the AsA untreated plants under stress. Ability of AsA to increase plant DM (Table 2) denying the adverse effects of DI stress, may have considerable contributions in improving plant growth and alleviating yield barrier arising from limited water conditions.

This coincides with our results that AsA foliar application considerably increased broccoli yields under DI stress, particularly under $20 \%$ loss of water requirements (Tables $3 \& 4$ ). The AsA is known to potentially generate a number of metabolic responses in plants, and affect a range of plant functions, including plant water relations Latif, et al. [46]. In this study, higher leaf RWC and MSI in broccoli plants treated with 1 or $2 \mathrm{mMAsA}$ could be maintained under DI compared with those in AsA-untreated plants (Table 5). These findings present AsA as a useful tool for improving DI stress tolerance in broccoli plants through enhancing the dehydration tolerance by increases of soluble sugars and proline as osmoprotectants (Table 6). Shinozaki, et al. [47] in this regard, reported that maintaining the integrity of cellular membranes under stress conditions is considered an integral part of drought tolerance mechanisms. The water status of broccoli leaves was expressed by RWC and WUE parameters (Tables 4 and 5). WUE, RWC and MSI were increased under water stress and further increased by AsA application. The increased RWC and MSI under DI stress by AsA application have maintained healthy metabolic processes in broccoli leaves. In this concern, Sinclair, et al. [48] reported that RWC considers a measure of plant water status, reflecting metabolic activity in plant tissues and used as a most meaningful index to identify the legumes with contrasting differences in dehydration tolerance. Abd El Mageed, et al. [10] concluded that there is a relationship between the RWC and the biomass (FM and DM) in plants under the interaction effect of DI stress and antioxidant application, which agreed with our study, indicating that RWC of broccoli leaves is basically dependent on the respective shoot biomass and suggesting that plants with greater biomass can maintain higher water content in their leaves; therefore, plants become more tolerant to DI stress. This parameter (RWC vs plant biomass or growth) could be used as a convenient evidence to distinguish the specific and non-specific traits for water deficit stress tolerance in broccoli plants.

Our results reported reductions in leaf photosynthetic pigment under DI stress conditions, however, AsA foliar spray increased total chlorophylls, carotenoids and anthocyanin concentrations in broccoli leaves under DI conditions when compared with DI-stressed plants without AsA (Table 5). These results are in conformity with those of Hussein, et al. [49] proving that AsA protects photosynthesis and enhances leaf photosynthetic pigments under drought, controlling the dry matter precipitation. In addition, the concentration of anthocyanin was increased with foliar application of AsA (Table 5), improving plant drought tolerance by stabilizing water potential as a proposed mechanism Tahkokorpi, et al. [50,51] reported that anthocyanin acts as scavenger of ROS through their hydrogen donating (antioxidant) potential. The increase in the concentration of carotenoids in broccoli plant with AsA application under DI stress also protects plants against the damaging effects of oxygen (exclusively bound to the core complexes of PSI and PSII) that is essential for chloroplast functioning. Also, it considers as an accessory pigment and an effective antioxidant, playing a unique role in protecting photochemical processes Burton et al. [52].

Osmotic adjustment, as an important mechanism, is included in plant adaptations to various stresses. Free proline and soluble sugars are major components of the osmoregulation in the expanded leaves of many species Mostajeran et al. [53]. Foliar application of AsA increased the concentrations of total soluble sugars, free proline and endogenous AsA in broccoli leaves under DI stress (Table 6). Thus, it was concluded that the AsA-induced increases in soluble sugars and proline concentrations under stress might contribute as a solute for the osmoregulation and/ or a substrate for the protein and polysaccharide syntheses in roots, and thereby for the growth of whole plants Sairam et al. [14]. Presence of endogenous AsA arising from AsA-pretreatment stimulated the various parameters in broccoli plants that can be explained as a result of AsA-enhanced growth or biomass of shoots under DI stress. Results of this study, therefore, prove that AsA may be effective for growth and related attributes under DI, and hence promote growth and leaf function. It has been concluded that, AsA is an important antioxidant that reacts with $\mathrm{H}_{2} \mathrm{O}_{2}, \mathrm{O}_{2} ; \mathrm{OH}^{-}$and lipid hydroperoxidases to repair their effects. In addition, AsA implicates in several types of biological activities in plants such as an enzyme co-factor, an antioxidant, and a donor/acceptor in electron transport at the plasma membrane or in the chloroplasts, all of which are related to oxidative stress resistance Conklin [54]. The biosynthesis of AsA from hexose phosphate and its involvement in protection against photo-oxidative stress suggest that there may be links between photosynthesis and the AsA pool size Reddy, et al. [55]. Moreover, the increase in the DPPH radical-scavenging activity in broccoli leaves grown under DI may be attributed to the increase of endogenous AsA concentration (Table 6) which suppressed free 
radicals by the formation of ascorbyl radicals Yamaguchi, et al. [56], which play an effective role in scavenging free radicals Pavet, et al. [57], generating more tolerance of broccoli plants to drought stress.

\section{Conclusion}

Subjecting broccoli plants to DI resulted in reductions in plant growth and yields, RWC, MSI, and leaf photosynthetic pigments, and increases in the concentrations of endogenous AsA, soluble sugars and proline, DPPH activity, and WUE. Totally, exogenous AsA application alleviated the adverse effects of DI stress on broccoli plants by further accumulation of soluble sugars and proline, and increasing RWC, acting as osmotic and metabolic regulators or substrates and in a part as cell component stabilizers. Besides, the further increase in DPPH activity and endogenous AsA, protecting the antioxidative defense system and increasing the tolerance of broccoli plants to DI stress. AsA may be involved in the upregulation of soluble sugars and proline biosynthesis for their promoting mechanism of drought tolerance in broccoli plants. This suggests that RWC might be dependent on water status of shoots arising from the increased levels of soluble sugars and proline in broccoli shoots, contributing to the maintenance of the water status in leaves of broccoli plants. This study recommends the use of AsA, at a rate of 1 or $2 \mathrm{mM}$, for improving growth and productivity, osmoprotectants and antioxidant activity in broccoli plants under DI by at least $20 \%$ of ETs.

\section{References}

1. Chun OK, Kim DO, Smith N, Schroeder D, Han JT (2005) Daily consumption of phenolics and total antioxidant capacity from fruit and vegetables in the American diet. Journal of the Science and Food and Agriculture 85(10): 1715-1724.

2. Wojciechowska R, Rozek S, Rydz A (2005) Broccoli yield and its quality in spring growing cycle as dependent on nitrogen fertilization. FoliaHorticulturae 17(2): 141-152.

3. Ngullie R, Biswas PK (2014) Performance of different varieties of broccoli under rainfed mid-hill conditions of Mokokchung district of Nagaland. International Journal of Farm Sciences 4(2): 76- 79.

4. Burt J, McKay A (1999) Broccoli production in Western Australia, Bulletin 4356, Agdex 254, May 1999, Agriculture Western, Australia.

5. Ludong DPM (2008) Effects of irrigation rate on the growth, yield, nutritive value, and water use efficiency of carrot (Daucus carota) and broccoli (Brassica oleracea). Ms. Thesis, Faculty of Agriculture, Curtin University of Technology, Australia.

6. World Bank (2006) Directions in Development. Reengaging in Agricultural Water Management: Challenges and Options. The International Bank for Reconstruction and Development/The World Bank, Washington, DC,1: 218.

7. Allen RG, Pereira LS, Raes D, Smith M (1998) Crop Evapotranspiration Guidelines for Computing CropWater Requirements. Irrigation and Drainage Paper 56. FAO, Rome, p. 300.

8. Fereres E, Soriano MA (2007) Deficit irrigation for reducing agricultural water use. Journal of Experimental Botany 58(2): 147-159.

9. Jones HG (2004) What is water use efficiency? In: Bacon, M.A. (Eds.), Water Use Efficiency in Plant Biology. Blackwell Publishing, Oxford, UK, p. 27-41.

10. Abd El Mageed TA, Semida WM, Mohamed GF, Rady MM (2016) Combined effect of foliar-applied salicylic acid and deficit irrigation on physiological-anatomical responses, and yield of squash plants under saline soil. South African Journal of Botany 106: 8-16.

11. Doorenbos J, Pruitt WO (1992) Crop water requirements. FAO Irrigation and Drainage. Paper No. 24, FAO, Rome, pp.144.

12. Batra NG, Sharma V, Kumari N (2014) Drought-induced changes in chlorophyll fluorescence, photosynthetic pigments, and thylakoid membrane proteins of Vigna radiate. Journal of Plant Interactions 1: 712-721.

13. Cruz De Carvalho MH (2008) Drought stress and reactive oxygen species: production, scavenging and signaling. Plant Signaling \& Behavior 3(3): 156-165.

14. Sairam RK, Deshmukh PS, Saxena DC (1998) Role of antioxidant systems in wheat genotype tolerance to water stress. Biologia Plantarum 41(3): 387-394.

15. Dolatabadian A, Modarressanavy SAM, Asilan KS (2010) Effect of ascorbic acid foliar application on yield, yield component and several morphological traits of grain corn under water deficit stress conditions. Notulae Scientia Biologicae 2(3): 45-50.

16. Hussein ZK, Khursheed MQ (2014) Effect of Foliar Application of Ascorbic Acid on Growth, Yield Components and Some Chemical Constituents of Wheat Under Water Stress Conditions. Jordan Journal of Agricultural Sciences, 10 (1): 1-15.

17. Page AI, Miller RH, Keeney DR (1982) Methods of Soil Analysis. Part 2: Chemical and Microbiological Properties. $\left(2^{\text {nd }} E d n\right)$ American Society of Agronomy Madison, Wisconsin, USA.

18. Klute A (1986) Methods of Soil Analysis. Part 1: Physical and Mineralogical Methods. ( $2^{\text {nd }}$ Edn), American Society of Agronomy Madison, Wisconsin, USA.

19. Israelsen OW, Hansen VE (1962) Irrigation principles and practices. Johan Wiley and Sonsins(Eds.) ( $3^{\text {rd }}$ Edn.). New York, USA.

20. Wallace OH, Munger HM (1965) Studies of the physiological basis for yield differences. 1. Growth analysis of six dry bean varieties. Crop Science 5: 343-348.

21. Hayat S, Ali B, Hasan SA, Ahmad A (2007) Brassinosteroid enhanced the level of antioxidants under cadmium stress in Brassica juncea. Environmental and Experimental Botany 60(1): 33-41.

22. Osman A Sh, Rady MM (2014) Effect of humic acid as an additive to growing media to enhance the production of eggplant and tomato transplants. The Journal of Horticultural Science and Biotechnology 89(3): 237-244

23. Rady MM (2011) Effect of 24-epibrassinolide on growth, yield, antioxidant system and cadmium content of bean (Phaseolus vulgaris L.) plants under salinity and cadmium stress. Scientia Horticulturae, 129(2): 232-237.

24. Jensen ME (1983) Design and Operation of Farm Irrigation Systems. ASAE, Michigan, USA, p. 827.

25. Arnon DI (1949) Copper enzymes in isolated chloroplasts. Polyphenoloxidase in Beta vulgaris L. Plant Physiology 24(1): 1-5.

26. Meyers KJ, Watkins CB, Pritts MP, HaiLiu R (2003) Antioxidant and antiproliferative activities of strawberries. Journal of Agricultural and Food Chemistry 51(23): 6887-6892.

27. Bates LS, Waldeen RP, Teare ID (1973) Rapid determination of free proline for water stress studies. Plant and Soil 39(1): 205-207.

28. Irigoyen JJ, Emerich DW, Sanchez Diaz M (1992) Water stress induced changes in the concentrations of proline and total soluble sugars in nodulated alfalfa (Medicago sativa) plants. Physiologia Plantarum 84(1): 455-460.

29. Lee SC, Kim JH, Jeong SM, Kim DR, Ha JU, et al. (2003) Effect of farinfrared radiation on the antioxidant activity of rice hulls. Journal of Agricultural and Food Chemistry 51(15): 4400-4403. 
30. Helrich K (1990) Official Methods of Analysis. Vitamin C (Ascorbic Acid). 15th Edition. Association of Official Analytical Chemists. Benjamin Franklin Station, Washington, DC, USA, pp. 1058-1059.

31. English M (1990) Deficit Irrigation. I: Analytical Framework. Journal of Irrigation and Drainage Engineering-ASCE 116(3): 399-412.

32. Loutfy N, El Tayeb, MA Hassanen AM, Moustafa MFM, Sakuma Y (2012) Changes in the water status and osmotic solute contents in response to drought and salicylic acid treatments in four different cultivars of wheat (Triticum aestivum). Journal of Plant Research 125(1): 173-184.

33. Mittler R (2002) Oxidative stress, antioxidants and stress tolerance. Trends in Plant Science 7(9): 405-410.

34. Athar HUR, Khan A, Ashraf M (2008) Exogenously applied ascorbic acid alleviates salt-induced oxidative stress in wheat. Environmental and Experimental Botany 63: 224-231.

35. Khan TA, Mazid M, Mohammad F (2011) Ascorbic acid: an enigmatic molecule to developmental and environmental stress in plant-A review. International Journal of applied Biology and Pharmaceutical Technology 2(3): 468-483.

36. Boutraa $\mathrm{T}$ (2010) Improvement of water use efficiency in irrigated agriculture: a review. Journal of Agronomy 9(1): 1-8.

37. Lawlor DW, Cornic G (2002) Photosynthetic carbon assimilation and associated metabolism in relation to water deficits in higher plants. Plant, Cell and Environment 25(2): 275-294.

38. Abdalla MM (2011) Beneficial effects of diatomite on the growth, the biochemical contents and polymorphic DNA in Lupinus albus plants grown under water stress. Agriculture and Biology Journal of North America 2: 207-220.

39. Azadeh R, Maryam F, Saeed S (2014) The effects of seed priming by ascorbic acid on some morphological and biochemical aspects of rapeseed (Brassica napus L.) under drought stress condition. International Journal of Biosciences 4(1): 432-442.

40. Zaicovski CB, Zimmerman T, Nora L, Nora FR, Silva JA, et al. (2008) Water stress increases cytokinin biosynthesis and delays postharvest yellowing of broccoli florets. Postharvest Biology and Technology 49(3): 436-439.

41. Erken O, Oztokat C (2010) Effects of Water Stress on Yield and Some Quality Parameters of Broccoli. In: 2nd International Symposium on Sustainable Development. Science book, pp. 231-237.

42. Malik S, Ashraf M, Arshad M, Malik TA (2015) Effect of ascorbic acid application on physiology of wheat under drought stress. Pakistan Journal of Agricultural Sciences 52(1): 209-217.

43. Elade Y (1992) The use of antioxidants to control gray mould (Botrytis cinerea) and white mould (Sclerotinia sclerotiorum) in various crops. Plant Pathology 141: 417-426.

ISSN: 2574-1241

DOI: 10.26717/BJSTR.2018.11.002031

Mostafa M Rady. Biomed J Sci \& Tech Res

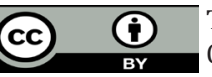

This work is licensed under Creative

Commons Attribution 4.0 License

Submission Link: https://biomedres.us/submit-manuscript.php
44. Navas P, Gomez Diaz C (1995) Ascorbate free radical and its role in growth control. Protoplasm, 184(1): 8-13.

45. Gupta PK, Nadgir AL, Macarentias AF, Jagannathan V (1980) Tissue culture of forest trees: Clonal multiplication of Tectona grandis L. (teak) by tissue culture. Plant Science Letters 17(3): 259-268.

46. Latif M, Akram NA, Ashraf M (2016) Regulation of some biochemical attributes in drought-stressed cauliflower (Brassica oleracea L.) by seed pre-treatment with ascorbic acid. The Journal of Horticultural Science and Biotechnology 91(2): 129-137.

47. Shinozaki K, Yamaguchi K (1997) Gene expression and signal transduction in water stress response. Plant Physiology 115(2): $327-$ 334.

48. Sinclair TR, Ludlow MM (1986) Influence of soil water supply on the plant water balance of four tropical grain legumes. Australian Journal of Plant Physiology 13(3): 329-341.

49. Hussein ZK, Khursheed MQ (2014) Effect of Foliar Application of Ascorbic Acid on Growth, Yield Components and Some Chemical Constituents of Wheat Under Water Stress Conditions. Jordan Journal of Agricultural Sciences, 10 (1): 1-15.

50. Tahkokorpi M (2010) Anthocyanins under drought and droughtrelated stresses in bilberry (Vaccinium myrtillus L.). Acta Universitatis Ouluensis: A Scientiae Rerum Naturalium 556: 1-46.

51. Syvacy A, Sokmen M (2004) Seasonal changes in antioxidant activity, total phenolic and anthocyanin constituent of the stems of two Morus species (Morus alba L. and Morus nigra L.). Plant Growth Regulation, 44(3): 251-256

52. Burton GW, Ingold KU (1984) Beta-carotene:an unusual type of lipid antioxidant. Science 224(4649): 569-573.

53. Mostajeran A, Rahimi Eichi V (2009) Effects of drought on growth and yield of rice (Oryza sativa L.) cultivars and accumulation of proline and soluble sugars in sheath and blades of their different age leaves. American-Eurasian Journal of Agricultural \& Environmental Sciences 5(2): 264-272.

54. Conklin PI (2001) Recent advances in the role and biosynthesis of ascorbic acid in plants. Plant, Cell and Environment 24(4): 383-394.

55. Reddy AR, Chaitanya KV, Vivekanandan M (2004) Drought-induced responses of photosynthesis and antioxidant metabolism in higher plants. Journal of Plant Physiology 161(11): 1189-1202.

56. Shinozaki K, Yamaguchi K (1997) Gene expression and signal transduction in water stress response. Plant Physiology 115(2): $327-$ 334.

57. Pavet V, Ollimos E, Kiddle G, Kumar S, Antonaiw J, et al. (2005) Ascorbic acid deficiency activates cell death and disease resistance in Arabidopsis thaliana. Plant Physiology 139(3): 1291-1303.

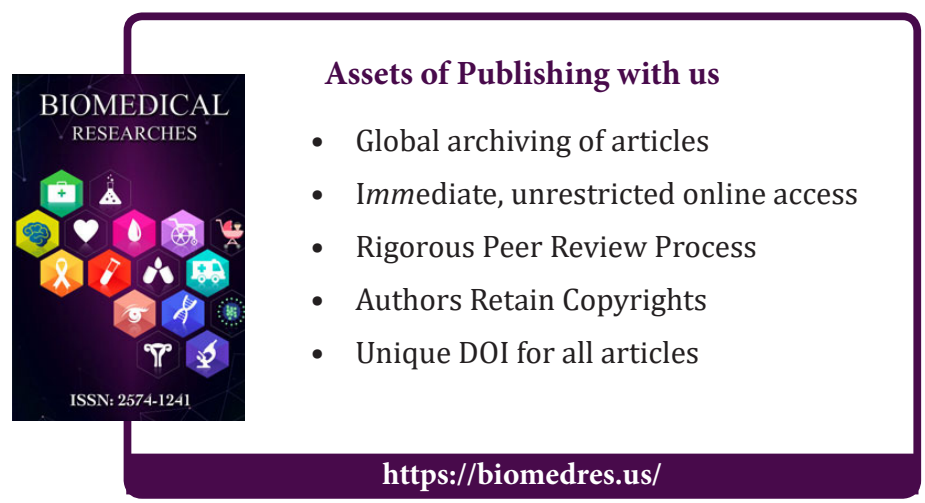

Cite this article: Ashraf Sh O, Mohamed H A W, Mostafa M R. Ascorbic Acid Improves Productivity, Physio Biochemical Attributes and Antioxidant Activity of Deficit Irrigated Broccoli Plants. Biomed J Sci\&Tech Res 11(1)-2018. BJSTR. MS.ID.002031. 